$57^{\text {ème }}$ Congrès de la SFMBCB, 03006 (2011)

DOI:10.1051/sfmbcb/20115703006

(C) Owned by the authors, published by EDP Sciences, 2011

\title{
Utilisation du rituximab chez les patients atteints de lésions linguales persistantes dues à un pemphigus vulgaire. Présentation de deux cas
}

\author{
Lerici $\mathrm{S}^{1}$, Boralevi $\mathrm{F}^{2}$, Catros $\mathrm{S}^{1}$, Fricain $\mathrm{JC}^{1}$ \\ ${ }^{1}$ UFR d'Odontologie, Pôle Odontologie et Santé buccale, CHU, Bordeaux, France \\ ${ }^{2}$ Service de Dermatologie, Hôpital des enfants, CHU, Bordeaux, France
}

Le pemphigus vulgaire se traduit par l'apparition de bulles intra-épithéliales. Dans sa forme cutanéo-muqueuse, la production prédominante d'anticorps anti-desmogléine 3 explique l'apparition précoce de lésions sur la muqueuse buccale (Anhalt 1982). Si les lésions buccales initiales sont bien connues, les lésions persistantes ont fait l'objet de peu d'études et leur traitement n'est pas codifié, contrairement à celui des lésions de la phase d'état traitées le plus souvent par une corticothérapie associée à des immunosuppresseurs (mycophénolate mofétil, ciclosporine, azathioprine, endoxan, méthotrexate...) pour permettre une épargne cortisonique (Bystryn 2005). Plus récemment un anticorps monoclonal anti-CD20, le rituximab, a été proposé pour traiter les pemphigus sévères cortico-résistants (Joly 2007).

Les cas rapportés sont ceux de deux patients, un homme de 47 ans et une femme de 72 ans, atteints d'un pemphigus vulgaire. Dans les deux cas, la pathologie a été confirmée par l'immunofluorescence directe de la muqueuse buccale et de la peau, et par l'immunofluorescence indirecte ; les tests ELISA sur sérum ont mis en évidence des anticorps anti-desmogléine 1 et 3 . Le premier patient était corticodépendant avec une recrudescence des lésions buccales pour des doses inférieures à $15 \mathrm{mg} / \mathrm{j}$ de prednisolone, malgré un traitement par mycophénolate mofétyl associé. Au moment de la consultation le patient était traité par cortancyl $(40 \mathrm{mg} / \mathrm{j})$ et mycophénolate mofétil $(2 \mathrm{~g} / \mathrm{j})$. Malgré ce traitement, il présentait une ulcération linguale granulomateuse, douloureuse, évoluant depuis plusieurs mois. La patiente était traitée par cortancyl $(1 \mathrm{mg} / \mathrm{kg})$ depuis un an avec une rémission des lésions cutanéomuqueuses à l'exception d'une lésion linguale douloureuse, persistant depuis plusieurs mois. Un traitement par rituximab a été introduit (4 injections à une semaine d'intervalle) à la posologie usuelle de $375 \mathrm{mg} / \mathrm{m} 2$ de surface corporelle. Il a permis une rémission complète des lésions persistantes chez les deux patients, mais également l'arrêt de la corticothérapie et du mycophénolate mofétil.

Le premier patient n'a pas récidivé 2 ans après l'arrêt du traitement par rituximab. La patiente, par contre, a présenté une récidive de ses lésions cutanées et buccales à 1 an après l'arrêt, ce qui a nécessité une nouvelle cure de rituximab.

Le rituximab est un anticorps monoclonal spécifique pour le CD20 humain, habituellement utilisé dans le traitement des lymphomes B. Le rôle des lymphocytes B dans les maladies auto-immunes a conduit à proposer son utilisation dans le traitement du pemphigus. L'efficacité du rituximab est basée sur l'hypothèse d'une altération des précurseurs de lymphocytes B produisant des anticorps anti-desmogléine 1 et 3 (Joly et al. 2007). La molécule a permis d'obtenir une disparition des lésions persistantes pour ces deux cas, mais elle n'a pas empêché la récidive pour un des patients. Ceci pose la question de son intérêt en traitement d'entretien, les risques de complications à long terme n'étant pas connus (Pfütze 2008).

Correspondance : seblerici@gmail.com 